\title{
El giro lingüístico hoy (o dónde fueron a parar los significados)
}

\author{
CRISTINA LAFONT \\ Department of Philosophy \\ Northwestern University \\ clafont@northwestern.edu
}

\begin{abstract}
Resumen: En este artículo analizo algunos hitos de la filosofía del lenguaje del siglo xx, tales como el surgimiento del externismo semántico en los años setenta y el auge del contextualismo en las últimas décadas, e intento mostrar cómo la lógica interna de dicha evolución deriva de la dificultad de mantener la noción fregeana de identidad de "pensamiento" o contenido proposicional. Desde esta perspectiva, el desarrollo del giro lingüístico en las últimas décadas hace pensar que la consecuencia lógica del intento fregeano de expulsar los pensamientos (o los significados) de la mente no puede ser otra que su eliminación.
\end{abstract}

Palabras clave: contextualismo, externismo semántico, identidad de contenido proposicional, literalismo

\begin{abstract}
In this article I analyze some milestones of the twentieth century philosophy of language such as the development of semantic externalism in the seventies and the revival of contextualism in recent decades. I seek to expose the internal logic of this evolution as a result of the difficulties for defending a Fregean notion of identity of "thought" or of propositional content. Seen from this perspective, the latest stages in the linguistic turn seem to indicate that the ultimate consequence of Frege's "extrusion of thoughts from the mind" can only be their elimination.
\end{abstract}

Key words: contextualism, identity of propositional content, literalism, semantic externalism

En su libro sobre los orígenes de la filosofía analítica, Michael Dummett identifica como paso decisivo hacia el giro lingüístico lo que él denomina "la expulsión de los pensamientos de la mente". Este paso fue dado no sólo por Frege, sino también por otros autores alemanes, como Bolzano, Meinong o Husserl, y, en general, por todos aquellos que participaron en la crítica al psicologismo en el periodo del cambio de siglo. Sin embargo, si consideramos la evolución del giro lingüístico a lo largo del siglo xx desde la perspectiva contemporánea, podríamos vernos tentados a replicar que Frege y Husserl no habían visto nada todavía, pues la verdadera expulsión de los pensamientos de la mente no tuvo lugar hasta los años setenta, aproximadamente, con el surgimiento de las teorías externistas del significado y el contenido conceptual. 
Para complicar la imagen más todavía, si atendemos a uno de los locus classicus del externismo semántico, "El significado de 'significado'" de Putnam, no deja de ser chocante que la tesis más desafiante a la concepción tradicional, a saber, que "los significados no están en la cabeza", se viera como un ataque a autores como Frege y, de hecho, formara parte de lo que se ha considerado una revolución antifregeana, a pesar de que para Frege la expulsión de los significados y los pensamientos de la mente fue uno de los objetivos fundamentales y recurrentes en sus escritos sobre cuestiones semánticas. Precisamente debido a las implicaciones cartesianas del psicologismo, Frege insistió en el carácter antiindividualista de los pensamientos, que "son susceptibles de ser propiedad común de muchos" (es decir, constituyen lo que Frege denominara el "tesoro común" que "se transmite de una generación a otra"), y en la necesidad de distinguirlos estrictamente de los contenidos de la mente individual, puramente subjetivos, que él denominó ideas (Vorstellungen). Más aún, lo que permitió a Frege sostener su crítica al psicologismo fue precisamente que logró articular un criterio decididamente antiindividualista para individuar dichos pensamientos mediante el recurso a la noción de verdad. Si el enunciado $P$ es verdadero y el enunciado $P^{\prime}$ es falso, $P$ y $P^{\prime}$ no expresan el mismo pensamiento. Según este criterio, los pensamientos están individuados por sus condiciones de verdad y éstas no son subjetivas o, como también podríamos decir, no están "en la cabeza". Eso explica por qué simplemente en virtud de entender las condiciones de verdad de un determinado enunciado todos podemos compartir el mismo pensamiento. Cualquier otra cosa que "esté en la cabeza" de un hablante sin estarlo en la de los demás es irrelevante para el pensamiento, no es un componente de éste, sino meramente una "idea" asociada con él. Desde esta perspectiva puede, sin duda, resultar extraño que el externismo semántico expresado en la tesis putnamiana de que "los significados no están en la cabeza" constituya uno de los ejemplos paradigmáticos de la revolución antifregeana de la filosofía del lenguaje de las últimas décadas.

Esta situación se puede entender mejor, quizás, si apelamos a otro criterio que Frege también ofreció para individuar pensamientos, el que suele llamarse criterio de significancia o valor cognoscitivo. Si los enunciados $P$ y $P^{\prime}$ no tienen el mismo valor cognoscitivo, es decir, si es posible para alguien que entiende $P$ y $P^{\prime}$ aceptar uno como verdadero y el otro no, entonces $P$ y $P^{\prime}$ no expresan el mismo pensamiento o, dicho en términos contemporáneos, no expresan el mismo contenido proposicional. La diferencia clave entre estos dos criterios radica en el hecho de que, aunque los dos hacen uso de la noción de verdad, el 
primero es puramente objetivo, mientras que el segundo tiene un claro componente subjetivo, es decir, en él se hace referencia a la noción de verdad, pero mediada por la situación epistémica particular del hablante. Dicho criterio depende esencialmente de la comprensión que de facto tenga un hablante particular de dichos enunciados, así como del resto de creencias, supuestos, asunciones, etc., que conjuntamente determinen su asentimiento o disentimiento. A diferencia de las condiciones de verdad, el valor cognoscitivo sí parece estar, en el sentido relevante, "en la cabeza" del hablante individual.

Esta noción internista de contenido cognoscitivo resultante del criterio de individuación fregeano basado en diferencias de actitud individual permite entender mejor que el externismo semántico haya estado íntimamente asociado a una revolución antifregeana en la filosofía del lenguaje reciente. Ello también explica el lugar central que ha ocupado en dicho debate la discusión en torno al fenómeno de las diferencias de valor cognoscitivo en la adscripción de actitudes proposicionales.

Esto último resulta especialmente obvio si se atiende a una determinada corriente del externismo semántico, a saber, la de las llamadas teorías de la referencia directa. ${ }^{1}$ Como es sabido, la "expulsión de los pensamientos de la mente" que propugna el externismo semántico radica en considerar que dichos pensamientos están individuados en parte por rasgos externos del entorno físico y social. Esto implica que la cuestión de si dos pensamientos expresan el mismo contenido o no, depende de factores del entorno que el hablante puede ignorar o, lo que es lo mismo, que no están necesariamente "en su mente". Las teorías de la referencia directa tienden a interpretar esta tesis externista o antiindividualista en el sentido de que los referentes mismos son constituyentes del pensamiento, es decir, del contenido proposicional expresado por los hablantes. Precisamente en la medida en que estas teorías incorporan la noción russelliana (o kaplaniana) de proposición singular, no resulta sorprendente que se hayan visto enfrentadas sistemáticamente a los famosos rompecabezas fregeanos relativos a las diferencias de valor cognoscitivo en la adscripción de actitudes proposicionales tales como la informatividad de enunciados de identidad y la insustituibilidad de términos correferenciales en contextos opacos.

Ahora bien, si se evalúa el externismo semántico desde esa perspectiva particular, podría parecer que las versiones neofregeanas de externismo $^{2}$ no tienen problemas con el criterio fregeano de diferencias de

${ }^{1}$ Ejemplos prominentes son K. Donnellan, S. Kripke, D. Kaplan, J. Perry y H. Putnam.

${ }^{2}$ Ejemplo prominentes son G. Evans, J. McDowell y C. Peacocke. 
valor cognoscitivo, pues en la medida en que éstas combinan la idea característicamente externista de pensamientos objeto-dependientes con la posibilidad de mantener los sentidos fregeanos, esto parece permitirles satisfacer el criterio de valor cognoscitivo. Sin embargo, esta impresión es engañosa, pues si la discusión de los últimos años es un buen indicador, las variantes neofregeanas y las antifregeanas parecen compartir igualmente, en cuanto teorías externistas o antiindividualistas, el cuestionamiento de la transparencia cognoscitiva de los pensamientos (es decir, el supuesto de que el hablante puede saber a priori si dos pensamientos tienen el mismo contenido o no). ${ }^{3}$ El mínimo común denominador de las diferentes variantes externistas o antiindividualistas parece radicar en lo que podríamos denominar el principio de opacidad de diferencias de contenido: un hablante puede tener dos pensamientos con distinto contenido en un momento determinado, a pesar de suponer que tienen el mismo contenido, y no ser capaz de darse cuenta de que tienen distinto contenido sin usar información empírica. ${ }^{4}$ Los ejemplos que apoyan este punto de vista apuntan a la ubicuidad de los fenómenos de deferencia y dominio imperfecto de conceptos en el uso de lenguajes naturales que el externismo ha puesto de manifiesto. Su forma habitual es la siguiente. ${ }^{5}$ Supongamos que un hablante está familiarizado con los términos "einstenio" y "fermio" y sabe que ambos nombran un elemento; sin embargo, no sabe que nombran dos elementos distintos ni conoce su estructura química. Ahora bien, ha tenido relaciones causales y/o deferenciales tales que cualquier antiindividualista aceptaría que tiene creencias en las que figuran los conceptos de dichos elementos. Por ejemplo, dicho hablante cree que einstenio es un elemento y que fermio es un elemento; sin embargo, no está seguro de si "einstenio" no es simplemente otro nombre para fermio (dado que muchos elementos con números atómicos altos han tenido dos nombres, normalmente originados en países distintos). Dado que sus creencias se refieren a elementos distintos, tienen de hecho contenidos

\footnotetext{
${ }^{3}$ Para una minuciosa defensa de esta tesis, véase Brown 2004. En la exposición y justificación de esta tesis que ofrezco a continuación me apoyo sustancialmente en la argumentación de Brown.

${ }^{4}$ En la expresión de este principio me he inspirado en el principio similar de opacidad de igualdad de contenido que Brown postula para las variantes de externismo social en Brown 1993 y los argumentos sobre la opacidad de diferencias de contenido subyacente a las teorías externistas neofregeanas que ofrece en Brown 2004.

${ }^{5}$ El ejemplo es de Frances 1999, p. 217, pero su aplicación para discutir los supuestos comunes a las versiones neofregeanas y no fregeanas de externismo es de Brown 2004.
} 
distintos. Pero el hablante podría creer que tienen el mismo contenido, en cuyo caso sólo empíricamente podría llegar a saber que no es así, es decir, mediante conocimiento empírico (sea sobre las clases naturales en cuestión o sobre las prácticas lingüísticas de su comunidad). ${ }^{6}$ Por tanto, el supuesto de transparencia de diferencias de contenido es falso.

Este tipo de ejemplos se puede generar con cualquier otra clase de términos (nombres propios, demostrativos, etc.) y se puede aplicar igualmente a pensamientos singulares demostrativos. Lo que hace interesante a este tipo de ejemplos es el hecho de que la estrategia fregeana de apelar a la noción de sentido no puede aplicarse en estos casos. En ejemplos de tipo kripkeano ${ }^{7}$ (en los que en las creencias de un hablante figuran dos conceptos con el mismo referente y contenido, pero el hablante no se da cuenta de que tienen el mismo contenido), el fregeano puede argüir que los dos conceptos difieren en sentido y, por tanto, en contenido, para defender el supuesto de transparencia de igualdad de contenido. Sin embargo, en los casos en los que en las creencias de un hablante figuran dos conceptos con referentes y contenidos distintos, sin que el hablante se dé cuenta de ello, la estrategia anterior no es viable. La noción de sentido se puede utilizar para introducir diferencias adicionales en los contenidos de los conceptos en cuestión, pero no para argumentar que dichos conceptos tienen el mismo contenido y así defender el supuesto de transparencia de diferencias de contenido.

$\mathrm{Si}$, como sugiere la discusión reciente en torno al externismo, se confirmara efectivamente la impresión de que los pensamientos o los contenidos proposicionales no son siempre transparentes para los propios hablantes, esto arrojaría una luz un tanto diferente respecto del verdadero impacto del externismo semántico en la filosofía del lenguaje contemporánea, pues sólo bajo el supuesto de que los pensamientos estaban individuados de modo perfectamente transparente para los propios hablantes tenía sentido postular dichos pensamientos como las unidades mínimas de significación que podían comunicarse entre hablantes y ser compartidas igualmente por todos ellos (es decir, ser el "tesoro común de pensamientos que se transmite de una generación a otra"). Si dicho supuesto no puede sostenerse, lo que se pone en cuestión es la viabilidad de postular contenidos proposicionales perfectamente in-

${ }^{6}$ El mismo tipo de ejemplo se puede generar no sólo con respecto al uso de términos, sino también con respecto a los referentes de los mismos. Por ejemplo, un hablante puede ser competente en el uso de los términos "olmo" y "haya", pero tener dudas sobre si los olmos y las hayas son, en realidad, la misma especie de árbol o no. Al respecto, véase Wettstein 2004, p. 136.

${ }^{7}$ Véase en Kripke 1979 el ejemplo original. 
dividuados, ${ }^{8}$ que son expresados lingüísticamente por los hablantes y transmitidos idénticamente de unos a otros, para explicar la comunicación lingüística. ${ }^{9}$ En ese caso, lo que tendría que rechazarse sería la inflación de la categoría misma de contenido proposicional para tales fines explicativos. ${ }^{10}$

Desde esa perspectiva, el debate entre los neofregeanos y los defensores de la referencia directa en torno a la explicación correcta de los elementos constituyentes del contenido proposicional puede que no haya sido más que un pequeño rodeo en el proceso abierto por el surgimiento del externismo semántico en general, ${ }^{11}$ pues dicho debate sugiere que el impacto de este último radicaría en la elaboración de una nueva concepción del contenido proposicional orientada a eliminar algunos supuestos internistas del modelo fregeano tradicional, precisamente en aras de completar "la expulsión de los pensamientos de la mente" iniciada por Frege. Sin embargo, si aceptamos con Dummett que Frege ya llevó a cabo dicha expulsión en el único sentido plausible en que esto puede hacerse, la verdadera contribución del externismo semántico parece radicar más bien en la consecuencia lógica de expulsar los pensamientos de la mente, es decir, en su eliminación.

Esta impresión parece confirmarse más allá de la discusión específica sobre externismo semántico si se tiene en cuenta el fuerte resurgimiento del contextualismo en la filosofía del lenguaje reciente. Sin duda, una contribución decisiva del externismo semántico desde el punto de vista del análisis del lenguaje en general ha sido mostrar la ubicuidad de la sensitividad al contexto en los lenguajes naturales. En esa medida, el externismo ha ofrecido, sin duda, apoyos adicionales a los defensores de una perspectiva pragmatista o contextualista en el análisis de la comunicación lingüística. Sin embargo, mi impresión es que su impacto en este contexto ha sido más profundo de lo que podría parecer a simple vista. Aunque en dicho terreno también puede observarse un debate irreconciliable entre los herederos de Frege (la semántica formal

${ }^{8} \mathrm{Si}$ tenemos en cuenta en este contexto el supuesto fregeano de que un concepto sin límites claros no es un concepto en absoluto, la prominencia que el debate sobre vaguedad ha adquirido en las últimas décadas podría verse como sintomática de la misma tendencia subyacente a la discusión en torno al externismo y al contextualismo en la filosofía del lenguaje contemporánea.

${ }^{9}$ La conexión entre el externismo semántico y el rechazo del modelo de la comunicación como transporte de contenidos idénticos de hablante a oyente es explícita y prominente en el caso de R. Brandom (véase 1994). También lo es en los argumentos que Wettstein presenta en su último libro (véase Wettstein 2004).

${ }^{10}$ Una detallada defensa de esta tesis puede encontrarse en Travis 2000.

${ }^{11}$ Para una detallada defensa de esta tesis véase Wettstein 2004. 
contemporánea que, siguiendo a Recanati, podríamos caracterizar de "literalista") y los herederos de la filosofía del lenguaje ordinario (los pragmatistas o "contextualistas", aliados más obvios del externismo), los términos de dicho debate parecen apuntar igualmente a la eliminación más que a la transformación de los "sentidos" o los "pensamientos" fregeanos.

En su reciente libro Literal Meaning, Recanati ofrece un análisis muy iluminador de los rasgos centrales de dicho debate. Básicamente, Recanati divide el espacio lógico del análisis del lenguaje contemporáneo en cinco posiciones situadas en una escala que va del extremo que él denomina "literalismo" al extremo opuesto que el denomina "contextualismo". De acuerdo con su análisis, sólo las versiones más extremas de este último constituyen un punto de vista eliminidor de los significados lingüísticos. Sin embargo, en nuestro contexto no está del todo claro que lo que las posiciones "literalistas" se niegan a eliminar por razones metodológicas equivalga realmente a los "pensamientos" fregeanos. Y, de ser así, tampoco estas posiciones ofrecerían una base firme contra la eliminación de estos últimos.

Para mostrar esto podemos circunscribir la disputa entre las posiciones analizadas por Recanati al criterio de individuación de pensamientos (o contenidos proposicionales) ofrecido por Frege en términos de condiciones de verdad. De acuerdo con este criterio, entender la proposición completa expresada por un enunciado consiste en entender sus condiciones de verdad. Cualquier otra cosa que el hablante dé a entender adicionalmente o implique con su enunciado no forma parte del contenido cognoscitivo del mismo en el sentido estricto del término, es decir, de lo evaluable en términos de condiciones de verdad.

Los literalistas aplican este criterio por la vía de distinguir, de entre todo aquello que un hablante puede dar a entender al expresar un enunciado en un contexto comunicativo determinado, la llamada proposición mínima, que sería, por tanto, el candidato más plausible a pensamiento fregeano, es decir, aquello compartido idénticamente por todos los hablantes en virtud de las características estrictamente lingüísticas de la oración utilizada y evaluable exclusivamente en términos de condiciones de verdad. En ese sentido, la proposición mínima equivaldría a lo que el enunciado expresa literalmente (en virtud de las propiedades semánticas de las expresiones tipo que la componen). Y, dado que esto último está controlado por las convenciones lingüísticas, es posible suponer que es entendido del mismo modo por todos los hablantes competentes. Por ello mismo, aunque lo dicho por el hablante 
vaya más allá de dicha proposición mínima y, en tales casos, sólo pueda entenderse completamente si se incorporan elementos contextuales adicionales, en la medida en que dicha incorporación está controlada por las convenciones lingüísticas, lo está del mismo modo para cualquier hablante y, en ese sentido al menos, su comprensión no es relativa al contexto. Para obtener dicha "proposición mínima" hay que imaginar el "contexto mínimo" en el que la oración en cuestión podría utilizarse para afirmar algo evaluable en términos de condiciones de verdad, es decir, una proposición completa.

Sin embargo, siguiendo este procedimiento se abre una brecha inevitable entre el significado lingüístico de la oración y el contenido proposicional o el "pensamiento" que el hablante quería expresar con ella, pues la "proposición mínima" no tiene por qué ser parte integral de lo aseverado por el hablante. ${ }^{12}$ Evidentemente, para entender el "pensamiento" que el hablante quiere comunicar, no basta con identificar una proposición completa posible. ${ }^{13}$ Lo que se requiere es identificar cuál proposición específica es la expresada por el hablante en un contexto determinado. Teniendo en cuenta semejante diferencia metodológica, sería una coincidencia verdaderamente asombrosa que procedimientos tan divergentes convergieran en sus resultados (de modo que los primeros pudieran considerarse una guía fiable para los segundos). Por eso pueden darse casos como, por ejemplo, los relativos al uso de descripciones definidas en los que el hablante utiliza una oración en un contexto para expresar un "pensamiento" singular sobre un determinado individuo (digamos, que el señor de la esquina lleva sombrero) y, sin embargo, la "proposición mínima" que la oración utilizada expresa literalmente es supuestamente una proposición general sobre el mundo (a saber, que existe una cosa u otra tal que es un señor, está en una esquina y lleva sombrero). Puesto que ambos "pensamientos" no tienen las mismas condiciones de verdad, siguiendo a Frege hay que concluir que, efectivamente, no son el mismo pensamiento.

\footnotetext{
${ }^{12}$ Véanse los ejemplos de Recanati 2004, pp. 8 y ss.

${ }^{13}$ Éste es especialmente el caso si se tiene en cuenta que lo que se pretende con este procedimiento es identificar la proposición completa "mínima", es decir, la más independiente del contexto posible, y, sin embargo, los hablantes pueden estar empeñados en comunicar "pensamientos" altamente sensitivos al contexto. No es de extrañar que, siguiendo dicho procedimiento, se llegue a la conclusión a la que llega Kent Bach, a saber, que "no hay nada particularmente normal en hablar literalmente. En efecto [. . .], la mayoría de las oraciones que se usan tienden a no usarse literalmente", ya que "un gran número de oraciones son muy difíciles de usar literalmente" (Bach 2005, pp. 28-29).
}

Diánoia, vol. LI, no. 56 (mayo 2006). 
El problema con este planteamiento no es tanto que el saber lingüístico no sea suficiente para identificar el "pensamiento completo" expresado por el hablante sin añadir saber del mundo contextual; el problema es más bien que dicho saber lingüístico así interpretado ${ }^{14}$ no parece ni siquiera necesario. Como parecen reconocer incluso sus defensores, la "proposición mínima" no coincide necesariamente con lo dicho por el hablante en el sentido fregeano del pensamiento completo (es decir, aquél que requiere conocer las condiciones de verdad específicas o el estado de cosas particular que tiene que ser el caso para que el enunciado sea verdadero). La proposición mínima no es necesariamente el pensamiento que el hablante quiere comunicar, ni siquiera es algo de lo que el hablante o los oyentes han de ser conscientes y, por tanto, tampoco es lo que intuitivamente entienden los hablantes. Si esto es así, esta estrategia metodológica no parece muy apropiada para alcanzar la meta fregeana de expulsar los pensamientos de la mente individual por la vía de identificar lo que está en la mente de todos los hablantes por igual, pues los "pensamientos" que identifica ni están en la mente de todos los hablantes ni son los que Frege quería expulsar. ${ }^{15}$

Con respecto a estos últimos, parece que tanto los literalistas como los contextualistas reconocen que su acceso e interpretación están esencialmente guiados por saber empírico sensitivo al contexto. Esto quiere decir que según cuáles sean las creencias, supuestos de fondo, asunciones, etc., de los diferentes hablantes, el potencial informativo de lo aseverado por cualquiera de ellos, así como su posible interpretación, variará necesariamente. Esto es precisamente lo que ponen de manifiesto los fenómenos de deferencia y dominio imperfecto de conceptos cuya ubicuidad el externismo semántico ha sacado a la luz. Ahora

\footnotetext{
${ }^{14}$ Evidentemente, los contextualistas no niegan un papel importante al saber lingüístico relativo a la sintaxis y al potencial semántico de los componentes de las oraciones para explicar la comunicación. Lo que niegan, en este contexto, es la necesidad (y plausibilidad) de atribuir adicionalmente un contenido proposicional a las oraciones (es decir, unas condiciones de verdad literales que éstas poseerían puramente en virtud de convenciones lingüísticas y, por tanto, independientemente del contexto) para explicar la comunicación. Como lo expresa Recanati, el significado literal de la oración, si es que existe tal cosa, no tiene privilegio composicional en el proceso de interpretación de las condiciones de verdad de lo dicho por los hablantes. Véanse Recanati 1995, y 2004, pp. 28 y ss.

${ }^{15}$ Resulta obvio añadir que la meta aquí es difícilmente opcional. Reconstruir lo que las oraciones supuestamente expresan "en sí mismas" puede resultar productivo para explicar lo que los hablantes expresan, o puede que no, pero sin duda la primera tarea no es inteligible con independencia de su posible utilidad para lograr la segunda.
} 
bien, si esto es así, las inevitables diferencias en saber empírico entre los hablantes ponen en cuestión la viabilidad de postular contenidos proposicionales idénticos y transparentes que son transmitidos de unos hablantes a otros para explicar la comunicación lingüística. El externismo semántico parece conducir naturalmente al rechazo de semejantes entidades intermedias entre los hablantes y aquello de lo que hablan. Vistas así las cosas, no es difícil anticipar que lo que el externismo acabe encontrando fuera de la mente no sean pensamientos fregeanos, sino simplemente el mundo de lo que hablamos. ${ }^{16}$

Ahora bien, una vez que hemos situado la discusión en torno al externismo en el ámbito de la filosofía del lenguaje en general, puede surgir la duda de si la estrategia de eliminitación llevada a sus últimas consecuencias no precipitaría al análisis del lenguaje y la comunicación a un escepticismo ${ }^{17}$ paralizador, forzado a intercambiar toda ambición explicativa por el dudoso consuelo de la filosofía como terapia. Puede que postular contenidos semánticos idénticos pertenecientes a las oraciones usadas por los hablantes no resulte una buena guía para explicar el potencial informativo de lo que los hablantes expresan o comunican con ellas; pero lo que es seguro es que eliminar toda guía contribuirá todavía menos a ofrecer tal explicación. Sin duda, las críticas a los intentos de articular una noción plausible y fructífera de identidad proposicional abundan. ${ }^{18}$ Lo que no abunda tanto son ejemplos constructivos de una estrategia alternativa que elimine todo rol explicativo para la noción de contenidos proposicionales idénticos transmitidos en la comunicación sin eliminar al mismo tiempo las ambiciones explicativas y sistemáticas a las que dicha noción debe su notoria permanencia. El externismo semántico ha mostrado, a lo sumo, la necesidad de una nueva estrategia metodológica; pero eso no es lo mismo que mostrar una alternativa viable y robusta de análisis del lenguaje para explicar la comunicación humana.

${ }^{16}$ Como lo expresa Wettstein en forma de objeción a Kaplan y Perry: de acuerdo con estos últimos, "las proposiciones están constituidas por lo que está del lado del mundo. Pero si tales proposiciones mundanas son prácticamente un oxímoron, dejemos de llamarlas proposiciones, y especialmente dejemos de llamarlas 'pensamientos', como hacen Kaplan y Perry algunas veces de modo confundente. Éstas son los correlatos mundanos de las oraciones, cuyos constituyentes no son representaciones de cosas y propiedades, sino las cosas y propiedades mismas" (Wettstein, 2004, p. 127; la traducción es mía).

${ }^{17} \mathrm{Al}$ respecto, véase el análisis del escepticismo semántico que ofrecen J. King y J. Stanley (2005).

${ }^{18}$ Ejemplos de críticas monográficas recientes son Travis 2000, y Wettstein 2004.

Diánoia, vol. LI, no. 56 (mayo 2006). 
En este contexto, no resulta difícil augurar que esta cuestión va a continuar siendo una herencia central del giro lingüístico inaugurado hace un siglo. Mas allá de esto, aventurarse a un pronóstico que tome partido por un lado u otro del debate sería embarcarse en el tipo de filosofía ficción característica de los periodos de cambio de siglo que, desgraciadamente, suele verse ampliamente superada por los acontecimientos. Sin embargo, no puedo resistir la tentación de apuntar al menos, antes de concluir, a lo que en mi opinión constituye un claro ejemplo reciente de combinación de altas ambiciones explicativas y sistemáticas (1) con una estrategia decididamente pragmatista y externista (2) que explícitamente renuncia a la noción de contenidos proposicionales idénticos transmitidos entre los hablantes para explicar la comunicación (3). Me refiero al modelo de scorekeeping desarrollado por Brandom en Making It Explicit para explicar nuestras prácticas comunicativas.

Lo extremadamente ambicioso del planteamiento de Brandom puede provocar serias dudas sobre sus perspectivas de éxito. Evidentemente, cuantas más cosas tienen que resultar correctas en una teoría, más improbable es el éxito de ésta. Y basta ojear Making It Explicit para darse cuenta de cuán numerosas son las que tienen que ser correctas en ese caso. Sin embargo, por eso mismo parece obvio que el planteamiento de Brandom puede ser criticado de todo, menos de falta de ambición explicativa. En esa medida, al menos es un ejemplo que desbanca la objeción más o menos tácita de los defensores de planteamientos tradicionales, según la cual adoptar un planteamiento decididamente pragmatista, contextualista y externista equivale necesariamente a renunciar al intento mismo de explicar la comunicación humana.

Efectivamente, está por verse si el intento de Brandom u otros intentos más moderados ${ }^{19}$ no resultarán totalmente fallidos; pero, sin duda, eso también está por verse en el caso de los planteamientos tradicionales. De hecho, algunos de éstos tienden a basar su estrategia de defensa más en argumentar por su supuesta superioridad metodológica, que en ofrecer ejemplos de análisis exitosos derivados de ellos. ${ }^{20}$ Lo que la proliferación de dichas defensas en la actualidad pone de relieve, sin embargo, es la seriedad del desafío que el externismo ha supuesto para la concepción del análisis del lenguaje tradicional. Vistas así las cosas,

${ }^{19}$ En comparación con el planteamiento de Brandon, planteamientos externistas como los de los defensores de la teoría de la referencia directa (Kaplan, Perry, Recanati, etc.), en la medida en que no renuncian abiertamente a la noción de identidad de contenido proposicional, pueden considerarse moderados.

${ }^{20}$ Un ejemplo característico es Fodor/Lepore (2002). 
es difícil aventurar dónde irán a parar los significados en este siglo. Ahora bien, que vuelvan a la "cabeza", es decir, que pierdan su carácter relacional, su dependencia del contexto (y, por tanto, también del saber empírico sobre el mundo desigualmente repartido entre los hablantes) por mor de mantener su quimérica identidad, parece ya inconcebible, incluso si limitamos la mirada a los planteamientos contemporáneos herederos del punto de vista tradicional.

\section{BIBLIOGRAFÍA}

Bach, K., 2005, "Context ex Machina", en Szabó 2005, pp. 15-44.

Brandom, R., 1994, Making It Explicit, Harvard University Press, Cambridge, Mass. [Versión en castellano: Hacerlo explícito. Razonamiento, representación y compromiso discursivo, trad. Ángela Ackermann y Joana Rosselló, Herder, Barcelona, 2005.]

Brown, J., 2004, Anti-Individualism and Knowledge, The MIT Press, Cambridge, Mass.

_ _ 1993, "Externalism and the Fregean Tradition", en A. Barber (comp.), Epistemology of Language, Oxford University Press, Oxford, pp. 431-458.

Burge, T., 1989, “Wherein is Language Social?", en A. George (comp.), Reflections on Chomsky, Blackwell, Oxford, pp. 175-191.

_ 1986 , "Intellectual Norms and Foundations of Mind", Journal of Philosophy, vol. 83, no. 12, pp. 697-720.

— 1979, "Individualism and the Mental", en P. French et al. (comps.), Midwest Studies in Philosophy, vol. IV, University of Minnesota Press, pp. 73-121. [Versión en castellano: "El individualismo y lo mental", trad. Salma Saab, en Margarita M. Valdés (comp.), Pensamiento y lenguaje. Problemas en la atribución de actitudes proposicionales, Instituto de Investigaciones FilosóficasUNAM, México, 1996, pp. 311-382.]

Donnellan, K., 1966, "Reference and Definite Descriptions," Philosophical Review, vol. 75, no. 3, pp. 281-304.

Dummett, M., 1994, Origins of Analytical Philosophy, Harvard University Press, Cambridge, Mass.

Evans, G., 1982, The Varieties of Reference, Oxford University Press, Oxford.

Fodor, J. y E. Lepore, 2002, The Compositionality Papers, Oxford University Press, Oxford.

Frances, B., 1999, "Contradictory Belief and Epistemic Closure", en Mind and Language, vol. 14, no. 2, pp. 203-226.

Frege, G., 1918, "Der Gedanke. Eine logische Untersuchung", en Logische Untersuchungen, ed. G. Patzig, Vandenhoeck und Ruprecht, Gotinga, 1986, pp. 30-53. [Versión en castellano: "El pensamiento: una investigación lógica", trad. Carlos Pereda, en Margarita M. Valdés (comp.), Pensamiento y lenguaje. Problemas en la atribución de actitudes proposicionales, Instituto de Investigaciones Filosóficas-UNAM, México, 1996, pp. 23-48.]

Diánoia, vol. LI, no. 56 (mayo 2006). 
Frege, G., 1892, "Über Sinn und Bedeutung”, en Funktion, Begriff, Bedeutung, ed. G. Patzig, Vandenhoeck und Ruprecht, Gotinga, 1980, pp. 40-65.

Kaplan, D., 1989, "Demonstratives", en J. Almong, J. Perry, H. Wettstein (comps.), Themes from Kaplan, Oxford University Press, Oxford.

King, J., y J. Stanley, 2005, "Semantics, Pragmatics, and the Role of Semantic Content”, en Szabó 2005, pp. 111-164.

Kripke, S., 1979, "A Puzzle about Belief", en A. Margalit (comp.), Meaning and Use, Dordrecht, Reidel, pp. 239-283.

_ 1980, Naming and Necessity, Harvard University Press, Cambridge, Mass. [Versión en castellano: El nombrar y la necesidad, 2a. ed., trad. Margarita M. Valdés, Instituto de Investigaciones Filosóficas-UnAM, México, 2005.]

McDowell, J., 1986, "Singular Thought and the Extent of Inner Space", Meaning, Knowledge and Reality, Harvard University Press, Cambridge, Mass., 1998, pp. 228-259.

Peacocke, C., 1983, Sense and Content, Oxford University Press, Oxford. Perry, J., 1979, “The Essential Indexical”, Noûs, vol. 13, no. 1, pp. 3-21.

Putnam, H., 1975, "The Meaning of 'Meaning'", Mind, Language and Reality, Philosophical Papers, vol. 2, Cambridge University Press, Cambridge, pp. 215-271. [Versión en castellano: El significado de "significado", trad. Jorge G. Flematti, Instituto de Investigaciones Filosóficas-UNAM, 1984.]

Recanati, F., 2004, Literal Meaning, Cambridge University Press, Cambridge, Mass.

_ 1995, "The Alleged Priority of Literal Interpretation”, en Cognitive Science, vol. 19, no. 2, pp. 207-232.

—, 1993, Direct Reference: From Language to Thought, Blackwell, Oxford.

Szabó, Z. (comp.), 2005, Semantics versus Pragmatics, Oxford University Press, Oxford.

Travis, C., 2000, Unshadowed Thought, Harvard University Press, Cambridge, Mass.

Wettstein, H., 2004, The Magic Prism, Oxford University Press, Oxford. , 1991, Has Semantics Rested on a Mistake? And Other Essays, Stanford University Press, Stanford.

Recibido el 8 de diciembre de 2005; aceptado el 26 de enero de 2006. 